\title{
Bidirectional gray matter changes after complex motor skill learning
}

\section{Martin Gryga, Marco Taubert, Juergen Dukart, Henning Vollmann, Virginia Conde, Bernhard Sehm, Arno Villringer and Patrick Ragert*}

Department of Neurology and Clinic for Cognitive Neurology, Max Planck Institute for Human Cognitive and Brain Sciences, University Hospital Leipzig, Leipzig, Germany

\section{Edited by:}

Neeraj Jain, National Brain Research

Centre, India

\section{Reviewed by:}

Preston E. Garraghty, Indiana

University, USA

Hugo Merchant, Universidad

Nacional Autónoma de México,

Mexico

Marc W. Haut, West Virginia

University School of Medicine, USA

*Correspondence:

Patrick Ragert, Department of

Neurology, Max Planck Institute for

Human Cognitive and Brain

Sciences, Stephanstrasse 1a,

04103 Leipzig, Germany.

e-mail: ragert@cbs.mpg.de
Long-term motor skill learning has been consistently shown to result in functional as well as structural changes in the adult human brain. However, the effect of short learning periods on brain structure is not well understood. In the present study, subjects performed a sequential pinch force task (SPFT) for 20 min on 5 consecutive days. Changes in brain structure were evaluated with anatomical magnetic resonance imaging (MRI) scans acquired on the first and last day of motor skill learning. Behaviorally, the SPFT resulted in sequence-specific learning with the trained (right) hand. Structural gray matter (GM) alterations in left $\mathrm{M} 1$, right ventral premotor cortex $(\mathrm{PMC})$ and right dorsolateral prefrontal cortex (DLPFC) correlated with performance improvements in the SPFT. More specifically we found that subjects with strong sequence-specific performance improvements in the SPFT also had larger increases in GM volume in the respective brain areas. On the other hand, subjects with small behavioral gains either showed no change or even a decrease in GM volume during the time course of learning. Furthermore, cerebellar GM volume before motor skill learning predicted $(A)$ individual learning-related changes in the SPFT and $(B)$ the amount of structural changes in left M1, right ventral PMC and DLPFC. In summary, we provide novel evidence that short-term motor skill learning is associated with learning-related structural brain alterations. Additionally, we showed that practicing a motor skill is not exclusively accompanied by increased GM volume. Instead, bidirectional structural alterations explained the variability of the individual learning success.

Keywords: motor learning, primary motor cortex (M1), dorsolateral prefrontal cortex (DLPFC), premotor cortex (PMC), magnetic resonance imaging (MRI)

\section{INTRODUCTION}

Complex motor skill learning over several weeks or years leads to structural changes in the adult human brain. In retrospective cross sectional studies, it has been shown that extensive years of practice in professional musicians was associated with gray matter (GM) volume differences in comparison to non-musicians (Gaser and Schlaug, 2003a,b). Subsequently, it was demonstrated in prospective investigations that just weeks or months of learning a three ball juggling cascade can induce structural brain changes in young normal volunteers (Draganski et al., 2004; Scholz et al., 2009) as well as in elderly subjects (Boyke et al., 2008). Recent findings provided compelling evidence that such use-dependent structural changes can be induced on a much shorter time scale usually within weeks of complex motor skill learning and that structural changes share dynamic properties comparable to those previously reported for functional brain changes (Filippi et al., 2010; Taubert et al., 2010). Additional confirmation about the behavioral relevance of structural changes as a consequence of complex motor skill learning comes from positive correlations between local GM volume changes and performance improvements over time (Taubert et al., 2010).

While these previous studies have qualitatively demonstrated that motor skill learning is accompanied by structural changes in the human brain, several important aspects have yet to be clarified: for example, it is unknown if structural alterations develop during the first week of training exposure and how they are related to individual performance improvements. Furthermore, the directionality of structural brain plasticity and its behavioral consequences has so far not been systematically investigated. While the majority of studies reported significant increases in GM as a consequence of skill learning (for review see Draganski and May, 2008), some studies also identified decreases in specific brain areas that are related to skilled performance (Stein et al., 2012; Taubert et al., 2010; Granert et al., 2011). Although the underlying cellular mechanisms of this learning-related bidirectional GM alterations still remain elusive these findings provide novel evidence that better performance is not exclusively linked with increased GM volume.

Based on the aforementioned studies, our primary hypothesis was that individual performance improvements in a complex motor task can be explained by individual GM alterations in M1 using a correlation analysis. Additionally, since previous studies have consistently shown that in addition to M1 also other brain areas are modulated by complex motor skill learning such as the cerebellum, premotor, and prefrontal areas (for review see 
Halsband and Lange, 2006) we also investigated structural brain changes using a whole brain exploratory analysis.

Another issue is the pronounced inter subject variability during motor learning. Based on previous findings (Tomassini et al., 2010) we hypothesized that individual (baseline) GM volume in task-related brain areas might predict individual performance improvements across the whole learning period as well as the extent of learning-induced structural brain changes.

\section{SUBJECTS, MATERIALS AND METHODS}

We studied 15 healthy subjects between 22 and 32 years of age (five females). All subjects gave written informed consent to participate in the experiment according to the declaration of Helsinki. The study was approved by the ethics committee of the University of Leipzig. Prior to participation, all subjects underwent a comprehensive neurological examination. They were not taking any medication. Subjects who did not meet the protocol criteria and/or had contraindications for the magnetic resonance imaging (MRI) measurements were excluded from participation. All subjects were task naïve. We did not include highly skilled subjects such as musicians, typists or sportsmen. According to the Oldfield questionnaire for the assessment of handedness (Oldfield, 1971), all subjects were right-handed [laterality score: $+100 \pm 12$ (median \pm stdev.) over a range of -100 (fully left-handed) and +100 (fully right-handed) where a value of 0 was used as a cut-off score between right and left handers].

\section{EXPERIMENTAL DESIGN}

The experiment comprised of five motor learning sessions on 5 consecutive days $(\mathrm{d} 1-\mathrm{d} 5)$. On each day, a sequential pinch force task (SPFT) was performed with the right hand for a total duration of 20 min with $5-30 \%$ of maximum voluntary contraction (MVC, $n=15)$. The time to perform the SPFT (20 min) on each day was motivated based on a previous motor learning study from our group (Vollmann et al., 2012).

In order to assess structural changes in the brain as a consequence of 5 days of motor skill learning, anatomical T1-weighted MRI scans were acquired $(n=13)$ on a 3 Tesla MRI scanner at the beginning (day 1) and the end of the experiment (day 5, see Figure 1).

\section{SEQUENTIAL PINCH FORCE TASK}

Subjects held a custom made pressure sensor between the thumb and index-finger of the right hand. During the SPFT, subjects had to continuously match the height of a moving reference bar (REF, right side of the screen) on a computer screen with another bar (FOR, left side of the screen) that visually displays their own pinch force generation between index-finger and thumb. For example, an increase in applied pressure caused an increase in the FOR bar. The force applied to the sensor was digitally sampled at $100 \mathrm{~Hz}$ and projected to the computer screen at a refresh rate of $60 \mathrm{~Hz}$. Subjects were asked to perform the task for a total duration of $16 \mathrm{~s}$ which included a sinusoidally varying sequence of force changes (learning sequence). This specific learning sequence was performed in 6 blocks per day (L1-6) separated by a 2 min resting period between each block. As a control for the sequence unrelated motor skill learning effect, we tested a random sequence at

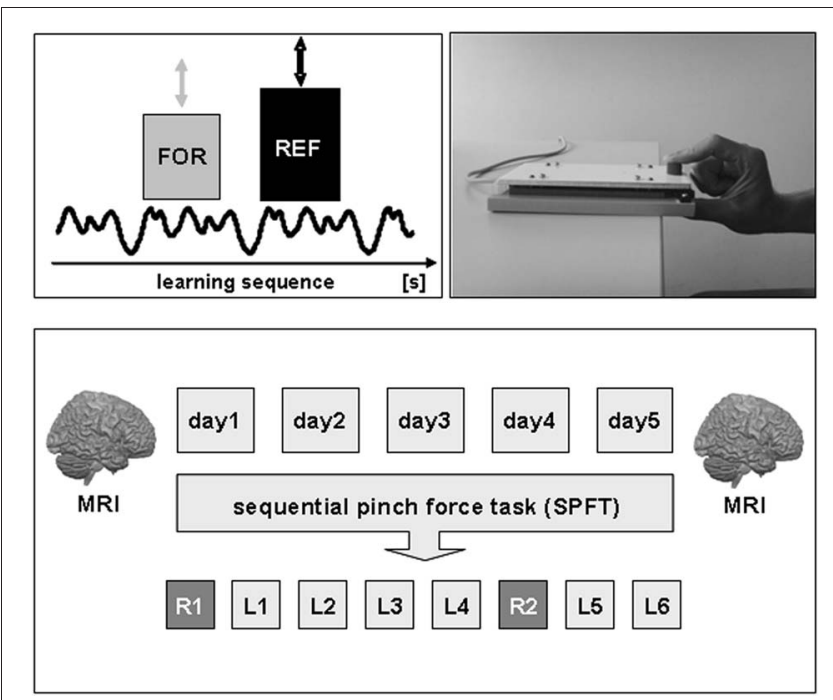

FIGURE 1 | Experimental setup and design. Subjects had to perform a sequential pinch force task (SPFT) with their right hand on 5 consecutive days (d1-d5). The SPFT consisted of a moving reference bar (REF), displayed on a computer screen, that had to be followed as exactly as possible with a force bar (FOR) that could be controlled by the individual force generation with the right hand. The SPFT was performed for a total duration of 20 min each day consisting of 2 random sequences (R1-2) as well as a learning sequence that was repeated six times (L1-6). In order to assess structural brain alterations as a consequence of 5 days of motor skill learning, anatomical T1-weighted MR images were acquired at the

beginning (day 1) and end of the experiment (day 5). For details see text.

the beginning (R1) as well as another random sequence after the fourth learning sequence (R2) of each practice session on each consecutive day (see Figure 1). Therefore, the learning sequence (L) was performed six times per day and the random sequence (R) two times per day. The duration, frequency and magnitude of force generation of the random sequence were matched to the learning sequence. The total time to perform the SPFT was approximately $20 \mathrm{~min}$ per day. Subjects were not aware about the difference between the sequences neither were they aware that the movement of the reference bar followed a specific sequence. During the task, electromyographic (EMG) activity of the left (control) and right (target) first dorsal interosseus (FDI) muscle was monitored online to ensure that the left FDI was at rest throughout the experiment. As a behavioral outcome measure for motor skill learning, we monitored the time lag (time difference in [ms]) between the FOR and REF bar continuously during the experiment. After each sequence (L or R) subjects received feedback about their individual performance (mean time lag [ms] per block) on a computer screen.

\section{STRUCTURAL MAGNETIC RESONANCE IMAGING ACQUISITION AND ANALYSIS}

MRI data was acquired on a 3 Tesla Magnetom Tim Trio scanner (Siemens, Erlangen, Germany) using a 32-channel head coil. We used the same protocol for each volunteer and each scanning session. On day 1 and 5, T1-weighted images were acquired using a MPRAGE sequence $\left(\mathrm{TR}=1.3 \mathrm{~s} ; \mathrm{TE}=3.46 \mathrm{~ms}\right.$; flip angle $=10^{\circ}$, 
FOV $=256 \times 240 \mathrm{~mm}$; 176 slices; voxel size $=1 \times 1 \times 1.5 \mathrm{~mm}$ ). The acquisition time for the anatomical MRI scan was approximately $13 \mathrm{~min}$.

All image processing steps and statistical analyses of MRI data were carried out using the SPM5 software package (Statistical Parametric Mapping software: http://www.fil.ion.ucl.ac.uk/spm/) implemented in Matlab 7.7 (MathWorks Inc., Sherborn, MA). All T1-weighted images were bias field corrected for inhomogeneity in order to obtain more uniform intensities within the different types of tissues. The T1-weighted MR images were processed using the DARTEL (Diffeomorphic Anatomical Registration Through Exponentiated Lie algebra) approach (Ashburner, 2007) to enable a more accurate spatial normalization. This approach includes a high dimensional warping procedure that provides an improved spatial normalization compared to other procedures implemented in SPM (Klein et al., 2009).

DARTEL registers all GM images to an averaged-size template created from all time points (day 1 and day 5 MRI scans) of all subjects used in this study. In order to preserve the total amount of signal, spatially normalized GM images were modulated. To transform these data to the MNI space the DARTEL averaged-size template was normalized to an a priori MNI template in SPM5 by using affine-only spatial normalization. The obtained normalization parameters were applied to the MR images before motor skill learning, day 1 (pre) and after motor skill learning, day 5 (post) of all subjects.

Subsequently, the images were smoothed using a Gaussian kernel of $8 \mathrm{~mm}$ full width at half maximum (FWHM). Additionally, the MR images were masked to avoid contamination by misclassified voxels. Voxels located between white matter (WM) and ventricular cerebrospinal fluid tends to be misclassified as GM voxels due to their similar intensity. The mask was obtained by excluding all voxels in the first and last template created by the DARTEL approach with a probability of below 0.2 (with a maximum value of one) for belonging to GM and including only the voxels that exceed this threshold in both templates. This mask was applied twice: (a) prior to smoothing to avoid misclassification, and (b), after smoothing to avoid edge effects.

First, we performed a paired $T$-test between GM volume before (day 1) and after (day 5) motor skill learning using a whole brain approach. To investigate potential correlations between structural changes and sequence-specific motor skill learning [change in learning sequence (day 1-5)/change in random sequence (day 1-5 [\%])], percent signal changes in GM volume were calculated for all voxels in the second MRI scan (day 5) using voxel intensities of the corresponding first MRI scan before learning (day 1) as baseline. Additionally, we performed the same correlation analysis for unspecific performance improvements in the random sequence (day 1-5 [\%]).

Percent signal change GM images were calculated for each single voxel in the brain of each subject using the following formula: $[G M($ day 5)/GM(day 1)] * 100. Percent signal change calculations were implemented in Matlab 7.7. For example, a value of 110 in a specific voxel would indicate that there is an increase of $10 \%$ in GM value from day 1 to day 5 in this specific voxel. Each structural MRI analysis was controlled for factors gender and age. Effects were reported for cluster of voxels exceeding a cluster size threshold of $p<0.05$ family wise error (FWE) corrected for multiple comparisons in the context of Gaussian random field theory and a voxel-level threshold of $p<0.001$ (uncorrected). The rationale for choosing this statistical threshold was motivated by the fact that a lot of the published morphometric studies investigating learning-induced GM plasticity used a comparable threshold approach (e.g., Ceccarelli et al., 2009; Taubert et al., 2010; Bezzola et al., 2011; Hoekzema et al, 2011). Hence, we wanted to be consistent with the literature to ensure comparability across studies. Since we had a strong a priori hypothesis about the involvement of M1 (BA 4) in complex motor tasks (Gaser and Schlaug, 2003a), we performed a small volume correction (FWE-corrected on voxel-level) for M1 $[(x=-37, y=-21, z=58$, MNI) according to a recent meta-analysis (Mayka et al., 2006)] using a $10 \mathrm{~mm}$ sphere.

To investigate whether the initial (baseline) GM volume predicts sequence-specific learning in the SPFT, we correlated GM volume of individual MR images on day 1 (before motor skill learning) with the amount of sequence-specific learning [\%] in the SPFT from day 1 to day 5 (see above). To test whether the amount of SPFT-induced structural changes is related to initial GM volume before motor skill learning, baseline GM volume was correlated with percentage changes in peak voxel intensities [first eigenvariate, SPM volume of interest (VOI) tool] of those brain regions that showed a significant relationship with sequence-specific improvements in the SPFT.

\section{STATISTICAL ANALYSIS}

Data were analyzed using PASW for Windows version 18. In order to identify sequence-specific changes in SPFT performance, we compared the last block/trial of the random sequence (R2) with the last block/trial of the learning sequence (L6) on each day using a $2 \times 5$ repeated measures ANOVA (ANOVARM) with factor TRIAL (R2, L6) and DAY (day 1-5). Subsequently, post hoc paired $T$-Tests were performed to compare sequence-specific versus unspecific procedural learning (Bonferroni-corrected for multiple comparisons) on each day. To relate motor learning to GM volume changes [\%] we used the following ratio for the correlation analysis: change in learning sequence (day 1-5)/change in random sequence (day 1-5). If necessary, a sphericity-correction (Greenhouse-Geisser correction) was performed for the respective statistical tests.

\section{RESULTS}

\section{MOTOR SKILL LEARNING IN A SEQUENTIAL PINCH FORCE TASK}

The absolute performance improvement in the SPFT of the learning sequence from the first (L1 on day 1: $246.67 \pm 14.01 \mathrm{~ms}$ ) to the last learning block (L6 on day 5: $103.33 \pm 11.37 \mathrm{~ms}$ ) was $59.92 \pm 5.66 \%$ (mean \pm s.e.m., see Figure 2A). Performing the random sequences (R1 and R2) on each day also resulted in significant performance improvements [R1 (day 1-5): $22.50 \pm 7.08 \%$; R2 (day 1-5): $27.23 \pm 5.62 \%$ ]. However, the amount of improvement in the learning sequence across days was higher as compared to both random sequences [L (1-30) vs. $\mathrm{R}_{1}(1-5)$ : $37.41 \pm 6.79 \%$; $\mathrm{L}(1-30)$ vs. $\mathrm{R}_{2}(1-5): 32.69 \pm 3.87 \%$; paired $T$-Tests: $\left.p<0.001\right]$.

ANOVA $_{\mathrm{RM}}$ with factor TRIAL (R2 and L6) and DAY (day 1-5) revealed a significant effect for factor TRIAL $\left[F_{(1,14)}=193.036\right.$; 


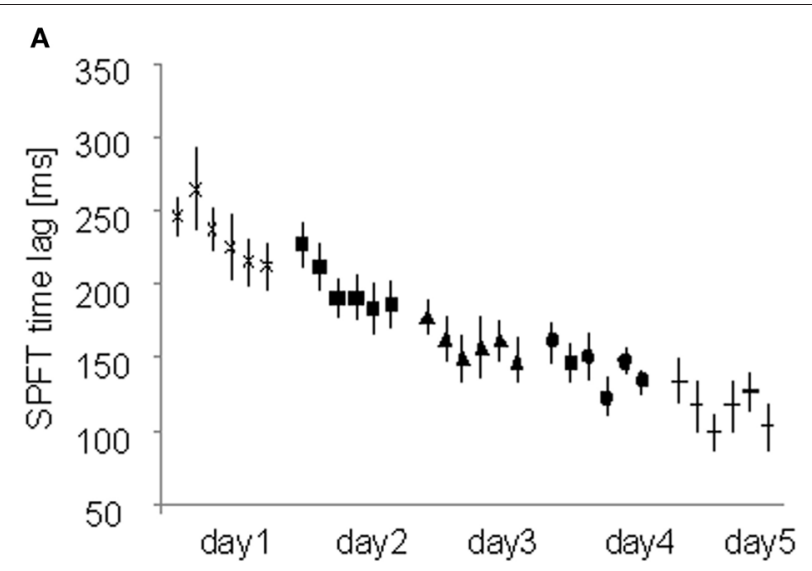

B

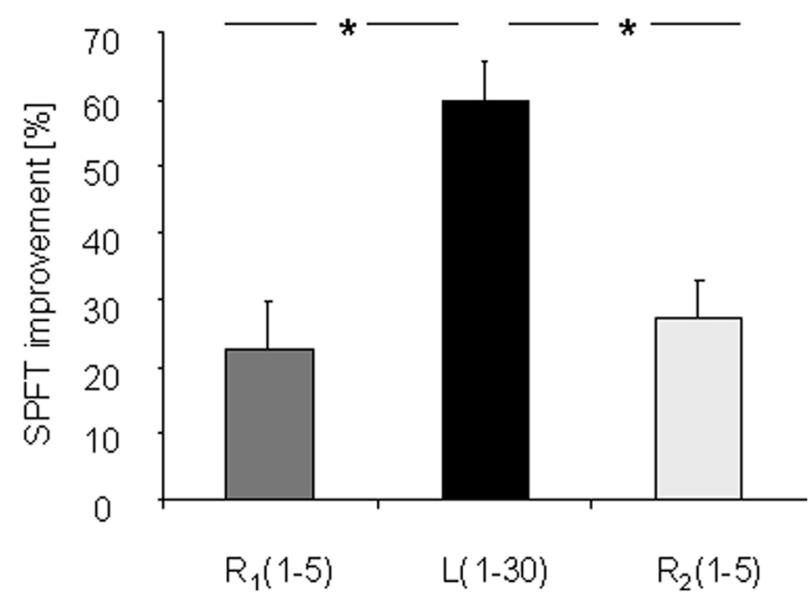

FIGURE 2 | (A) Absolute performance improvements [ms] for the learning $(\mathrm{L})$ and random FEW sequence in the SPFT on 5 consecutive days (day 1-5). (B) Procedural sequence-specific learning over 5 consecutive days with the right hand. Procedural sequence-specific learning was expressed as the difference between the last trial of the random sequence (R2) versus the last trial of the learning sequence (L6) on each day. *Indicates significant performance changes [Bonferroni-corrected for multiple comparisons $(p \leq 0.035)$ ].

$p<0.001]$ and DAY $\left[F_{(4,56)}=27.447 ; p<0.001\right]$ as well as a significant interaction between TRIAL and DAY $\left[F_{(4,56)}=4.829\right.$; $p=0.002$ ] indicating significant procedural sequence-specific learning within each day and over 5 consecutive days with the right hand (see Figure 2B). Post-hoc comparisons (Bonferronicorrected) revealed a significant increase in procedural sequencespecific learning from day 1 to day $5(p=0.035)$ and from day 2 to day $5(p=0.004)$ while other comparisons remained non-significant $(p>0.05$, see Figure 2B).

In an additional analysis, we investigated whether the newly acquired motor skill in the SPFT consolidated across days. Therefore, we compared performance of the last learning sequence on each day with the initial performance of the same sequence on the next day. No significant difference was observed between days (day 1 vs. day $2 p=0.12$; day 2 vs. day $3: p=0.37$; day 3 vs. day $4: p=0.25$; day 4 vs. day $5: p=0.99$ ) indicating successful retention of the newly acquired skill to the next day without further off-line improvements.

\section{STRUCTURAL BRAIN CHANGES AS A CONSEQUENCE OF MOTOR SKILL LEARNING}

Comparing differences in mean GM volume between day 1 and day 5 revealed no significant effect over time $(p<0.05$, whole brain analysis). There are two possibilities that might explain the lack of significance over time when using a paired-T-Test: (A) Five days of motor skill learning in general were not sufficient to drive structural brain reorganization or (B) some subjects showed an increase GM volume while others showed a decrease which as a consequence did not translate into statistically detectable difference of the mean GM volume. However, it is reasonable to assume that especially these bidirectional brain alterations (either local increases or decreases in GM volume) might still have a strong impact on inter-individual differences motor skill learning (for review see Kanai and Rees, 2011). Indeed, based on our behavioral results, the amount of motor skill learning was highly variable across subjects. To account for this individual variance, we subsequently performed a linear correlation analysis between individual sequence-specific performance improvements in the SPFT and changes in GM volume (day 5 vs. day 1 [\%]). The correlation revealed a significant positive linear relationship in the left M1 covering the hand area (BA4, $r=0.841, p<0.0001$ ) as well as in the right ventral premotor cortex [ventral PMC, according to (Mayka et al., 2006), BA6, $r=0.941, p<0.001$ ] and dorsolateral prefrontal cortex (DLPFC, BA9; $r=0.83, p<$ 0.0001 , see also Figure 3B and Table 1). These results indicate that most of the subjects with larger sequence-specific performance improvements in the SPFT were those with larger increases in GM volume in the respective brain areas. On the other hand, subjects with only little behavioral gains were generally those that showed either a weak increase or even a decrease in GM volume. To further support this claim, we subdivided our subjects into good $(n=6)$ and poor learners $(n=7)$ according to the group mean of sequence-specific changes in SPFT performance [SPFT improvement $27 \%$ (group mean); where subjects $>27 \%$ are defined as good learners and subjects $<27 \%$ are defined as poor learners] and compared the GM volume changes across subgroups and brain regions (left $\mathrm{M} 1$, right ventral PMC and DLPFC). On the one hand, poor learners showed a non-significant trend toward a learning-related GM volume reductions in left M1 as compared to good learners $(-1.85 \pm 1.75 \%$ vs. $3.20 \pm 2.15 \%$, MannWhitney $U$-Test: $p=0.138)$. On the other hand, good learners showed significant larger GM volume changes in right ventral PMC and DLPFC as compared to poor learners (ventral PMC: $3.00 \pm 1.46 \%$ vs. $-0.58 \pm 1.75 \%$, Mann-Whitney $U$-Test: $p=$ 0.014 ; DLPFC: $1.31 \pm 0.53 \%$ vs. $-1.14 \pm 0.70 \%$, Mann-Whitney $U$-Test: $p=0.008$ ).

Sequence-unspecific performance improvement in the random sequence was also associated with GM volume changes in right DLPFC (BA9) and left (BA8) superior frontal gyrus (SFG), right middle frontal gyrus (MTG, BA8), left dorsal premotor cortex (dorsal PMC, BA6) as well as in left inferior frontal gyrus (IFG, BA44, see Table 1). These results indicate distinct and divergent 


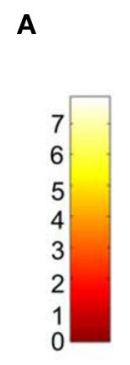

T-score

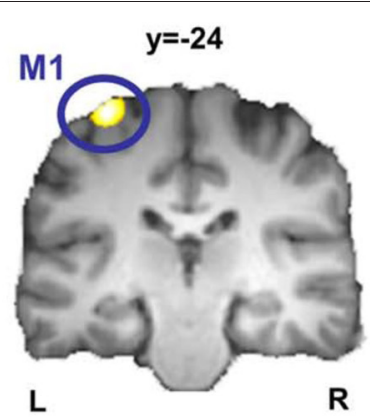

$\mathbf{R}$

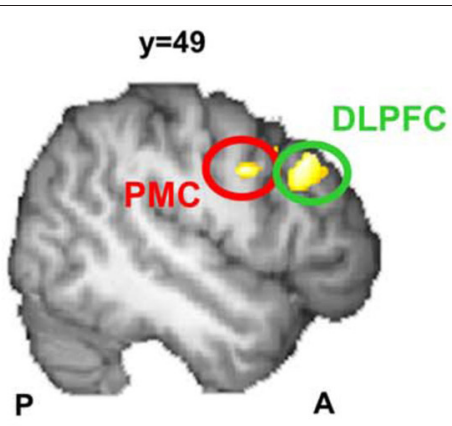

A

B
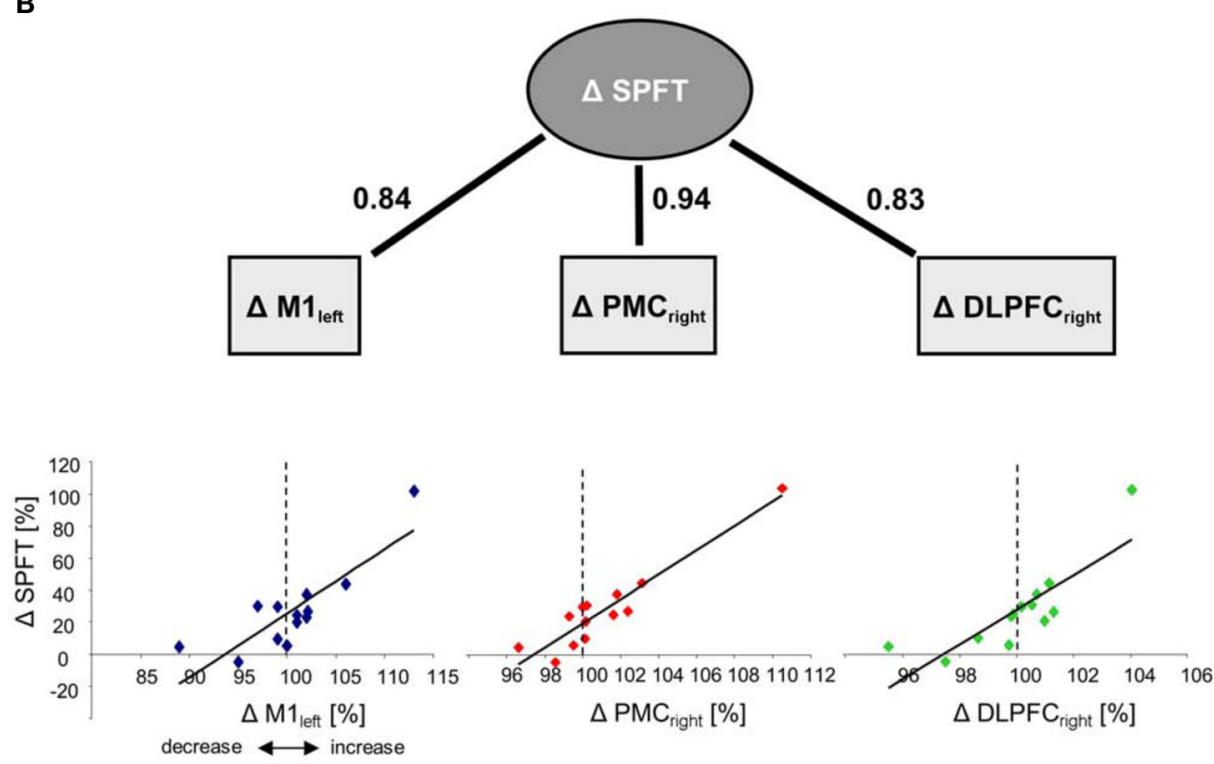

FIGURE 3 | Correlation analyses between GM volume changes and sequence-specific performance improvements in SPFT [change in learning sequence (day 1-5)/change in random sequence (day 1-5 [\%])]. Correlations are displayed on a structural template constructed with DARTEL. All images are shown at $p<0.001$ with an extend threshold of $k=800$ voxels for display purpose only ( $p<0.05$ FWE correction on cluster level). Bars indicate $t$-values. (A) Significant correlation between GM volume changes (day 5 vs. day 1 [\%]) in left primary motor cortex (M1), right premotor cortex (PMC) and dorsolateral prefrontal cortex (DLPFC) and sequence-specific performance improvements [\%] in the SPFT. Please note that the subject with the highest change in GM and SPFT is the same across the three brain areas (left M1, right ventral PMC, and right DLPFC) (B). Solid lines indicate significant correlations (where numbers indicate the respective $r$-values). $\Delta$ represents \% signal changes in GM or performance improvements in the SPFT. L, left; $R$, right; $P$, posterior; A, anterior. structural brain alterations as a consequence of learning-specific and unspecific performance improvements in the SPFT.

In a further step we asked whether initial GM volume might predict the outcome of sequence-specific motor learning across days. We found that initial GM volume in bilateral cerebellum (right: cerebellar lobule VI, left: cerebellar crus2) and right middle temporal gyrus (MTG, BA21) correlated positively with the sequence-specific motor learning over 5 consecutive days (see also Figure 4A as well as Table 2). These results indicate that the initial GM volume in specific cortical networks might serve as a predictor for successful sequence-specific motor skill learning. Even more interestingly, GM volume in bilateral cerebellum (right: cerebellar lobule VI, left: cerebellar crus2) predicted the amount of structural GM changes (GM volume changes [\%]) after sequence-specific motor learning in left $\mathrm{M} 1$, right ventral PMC and DLPFC (Figure 4B and Table 3).

\section{DISCUSSION}

In the present study we confirmed previous work showing that skill learning is associated with GM volume changes that are directly linked with individual performance improvements. However, we here extended these findings by providing novel evidence that the individual learning rate is associated not only with increased GM volume but also with bidirectional structural changes. More specifically, we showed that the individual learning success in a SPFT after $5 \times 20 \mathrm{~min}$ of SPFT training (day 1-5) positively correlated with structural GM volume changes in left M1, right ventral PMC and DLPFC. Interestingly, most of the subjects with large behavioral gains were those that showed learning-related GM volume increases while some subjects with only little performance improvements either showed no change or a decreased GM volume in the respective brain areas. Furthermore, the initial (baseline) GM volume of bilateral 
Table 1 | Positive correlation between GM volume changes and (A) sequence-specific motor skill learning in a SPFT as well as (B) sequence-unspecific performance improvements (day 5 vs. day 1 [\%]).

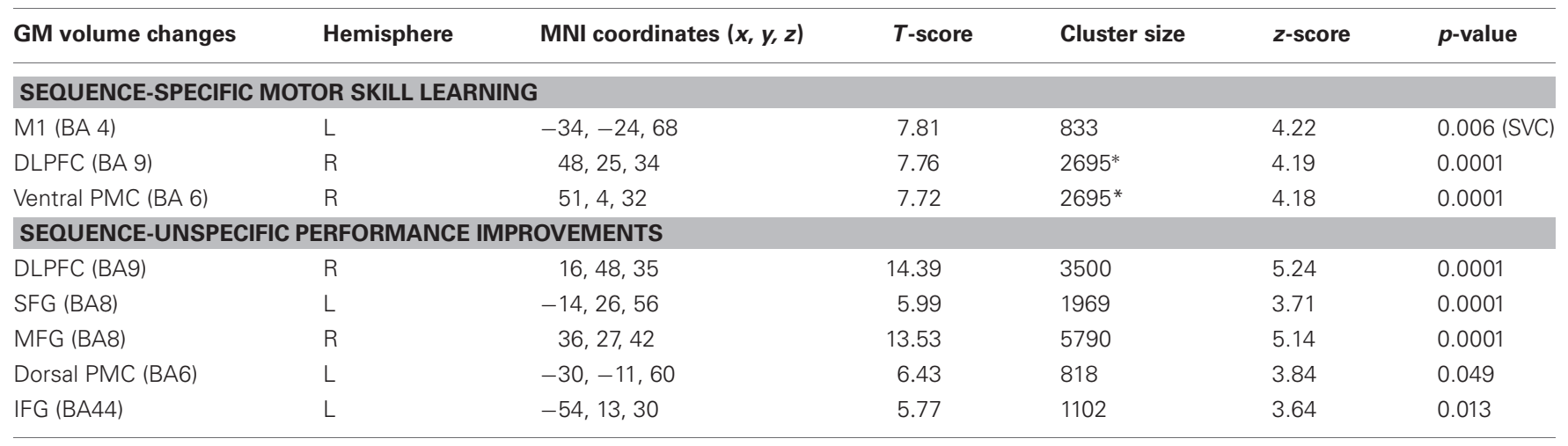

$L$, left; $R$, right; SVC, small volume correction.

$p$-values represent results with non-stationary correction on a cluster-level ( $p<0.05$, FWE-corrected).

* Indicates presence of sub-clusters within one cluster.

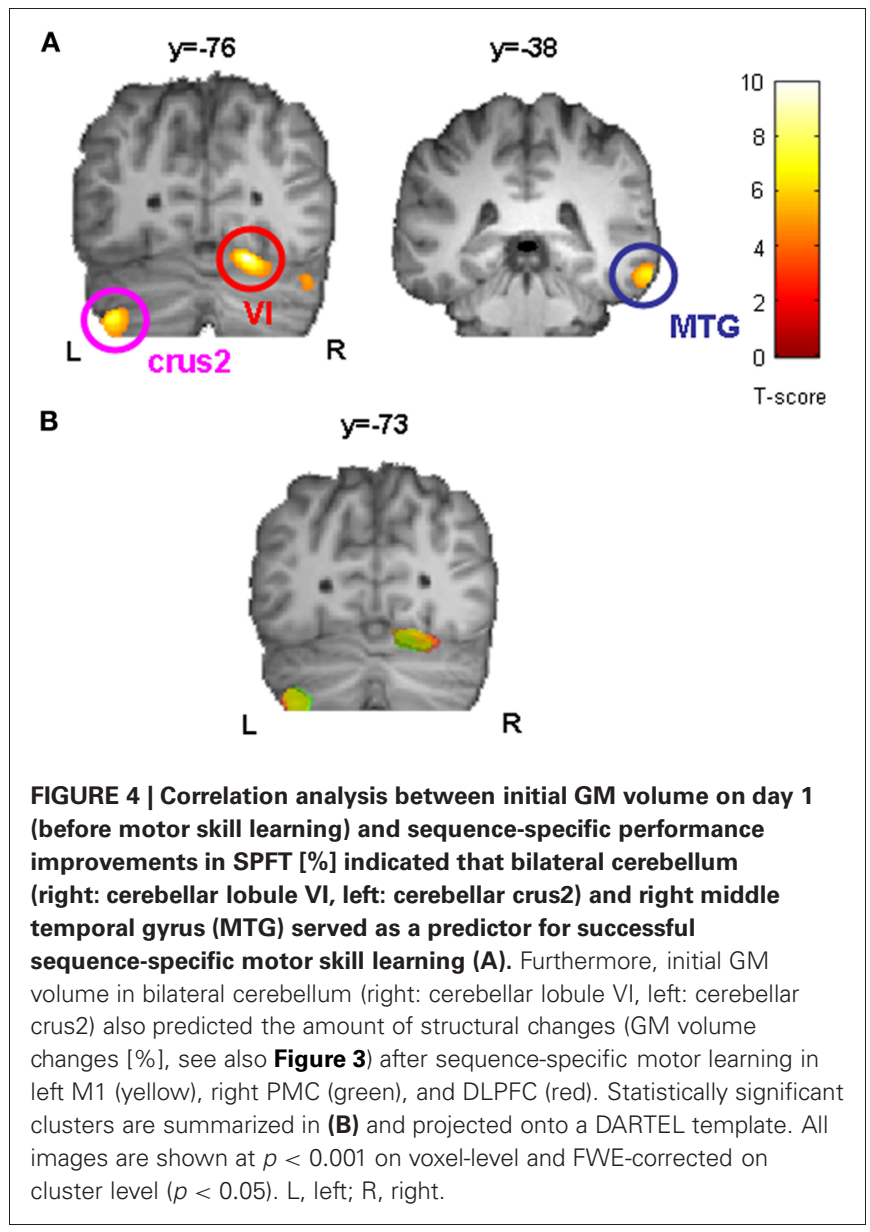

cerebellum positively correlated with sequence-specific motor learning and structural changes across days. Hence, initial differences in cerebellar GM volume seems to "predict" whether subjects will show improved performance with training and whether there will be training-related changes in cerebral cortical
GM. It seems quite likely that initial differences in cerebellar GM volume reflect important differences in the subjects' motor learning histories prior to their involvement in this experiment. In this view, subjects might have started the experiment at different locations along some hypothetical motor learning continuum, with the initial cerebellar measurements serving as the index of their pre-training potential for behavioral improvement and correlated brain changes. However, it is important to keep in mind that in our study, subjects with a history of enhanced (hand) use (such as musicians and sportsmen) were excluded from participation. Thus, it is likely that other factors such as genetic predisposition, synaptic history before training or other unknown determinants might as well account for individual differences in motor learning across subjects.

To our knowledge, this is the first study demonstrating fast evolving structural brain changes as early as after 5 consecutive days of SPFT learning that correlated with the individual learning rate. Similar fast evolving structural alterations were previously observed after daily application of low-frequency repetitive transcranial magnetic stimulation (May et al., 2007) or complex motor skill adaptation (Landi et al., 2011). In a similar vein, using a neuropharmacological approach, it has been shown recently that a single application of haloperidol, a $\mathrm{D}_{2}$ receptor blocker, is capable of evoking significant structural alterations in bilateral putamen within hours after intake (Tost et al., 2010). Likewise, a recent study provided evidence that learning to juggle (a complex multimodal task) seems to alter GM volume in the occipitotemporal cortex as early as after 7 days of training even though this was a non-significant trend only. Structural changes in the above mentioned study were not related to the individual performance improvements (Driemeyer et al., 2008). Progressive (dynamic) morphological alterations in gray and white matter were observed during learning a whole body balancing task for several weeks and in that study, individual performance improvements directly correlated with structural gray and white matter changes after six consecutive weeks of learning (Taubert et al., 2010). 
Table 2 | Initial (baseline) GM volume predicts the amount of sequence-specific motor skill learning in a SPFT.

\begin{tabular}{|c|c|c|c|c|c|c|}
\hline GM volume & Hemisphere & MNI coordinates $(x, y, z)$ & $T$-score & Cluster size & $z$-score & $p$-value \\
\hline Cerebellum (lobule VI) & $\mathrm{R}$ & $18,-76,-17$ & 9.96 & 3145 & 4.63 & 0.0001 \\
\hline Cerebellum (crus 2) & $\mathrm{L}$ & $-40,-72,-43$ & 9.93 & 1768 & 4.62 & 0.002 \\
\hline
\end{tabular}

$L$, left; $R$, right.

$p$-values represent results with non-stationary correction on a cluster-level $(p<0.05$, FWE-corrected).

Table 3 | Initial (baseline) GM volume predicts the amount of GM volume changes that correlated with SPFT improvements (see also Table 1).

\begin{tabular}{|c|c|c|c|c|c|c|}
\hline GM volume & Hemisphere & MNI coordinates $(x, y, z)$ & $T$-score & Cluster size & z-score & $p$-value \\
\hline \multicolumn{7}{|c|}{ PREDICTOR RIGHT DLPFC } \\
\hline Cerebellum (lobule VI) & $\mathrm{R}$ & $19,-73,-17$ & 12.20 & 3735 & 4.97 & 0.0001 \\
\hline \multicolumn{7}{|l|}{ PREDICTOR LEFT M1 } \\
\hline Cerebellum (lobule VI) & $\mathrm{R}$ & $19,-71,-14$ & 12.78 & 3138 & 5.05 & 0.0001 \\
\hline \multicolumn{7}{|c|}{ PREDICTOR RIGHT PMC } \\
\hline Cerebellum (lobule VI) & $\mathrm{R}$ & $17,-78,-19$ & 10.38 & 3058 & 4.70 & 0.0001 \\
\hline Cerebellum (crus 2) & $\mathrm{L}$ & $-36,-73,-45$ & 6.06 & 1462 & 3.74 & 0.010 \\
\hline
\end{tabular}

$L$, left; $R$, right.

$p$-values represent results with non-stationary correction on a cluster-level $(p<0.05$, FWE-corrected).

We found a positive linear relationship in several motorrelated brain regions including the left M1 (BA 4), the right ventral premotor cortex (ventral PMC, BA6) as well as the right dorsolateral prefrontal cortex (DLPFC, BA 9). This finding is in line with recent functional MRI studies showing that the individual positive BOLD signal change was associated with improved task performance (Floyer-Lea and Matthews, 2004; Tegenthoff et al., 2005). For example, in a similar pinch force task, a significant correlation between BOLD signal changes in M1 and DLPFC including middle frontal gyrus and improvements in task performance were observed during short-term motor skill learning (Floyer-Lea and Matthews, 2004). Activation of the (right) DLPFC is commonly reported during complex visuomotor learning tasks (Jenkins et al., 1994; Fink et al., 1999; Toni and Passingham, 1999) and might serve as a temporal storage region of modality specific sensorimotor associations (for review see Halsband and Lange, 2006).

Several previous studies suggested that PMC has a major role in motor learning and control of sequential movements (Schubotz and von Cramon, 2001a,b). Activation in PMC during motor skill learning has been usually observed bilaterally. While activation of the right PMC reflects spatial processing (e.g., transformation of visual signals into an appropriate motor output), which has been hypothesized to be important during the initial phase of motor learning, activation of the left PMC seems to be involved in later phases of motor learning (Ghilardi et al., 2000). Furthermore, it has been shown that the right (ventral) PMC is involved in processes linked to sequential motor actions and might, therefore, have an important role in sequence generation from memory (Tanaka et al., 2009). This is in good accordance with a previous study showing that the right PMC has a role in working memory (Sadato et al., 1996).

Taken together our findings support the hypothesis that even subtle and fast evolving bidirectional structural brain alterations in left M1, right ventral PMC and right DLPFC appear to have a crucial role in specific aspects of complex motor skill learning. Similar fast evolving structural changes have also been observed in animals performing a complex motor learning task. More specifically, motor practice over 2 days resulted in significant spine remodeling that correlated with behavioral improvements after learning (Yang et al., 2009).

We did not detect any learning-related structural changes in the cerebellum which was shown to be involved in functional MRI studies (Debaere et al., 2004; Floyer-Lea and Matthews, 2004, 2005) and lesion studies (Werner et al., 2010) and has been suggested to be a crucial mediator for fine motor control and motor learning (Jueptner et al., 1997; Jueptner and Weiller, 1998; Hikosaka et al., 2002; Halsband and Lange, 2006; Ramnani, 2006). In the present study, the lack of learning-related structural alterations in the cerebellum was unexpected and certainly requires further investigation. However, we believe the learning-related functional changes in the cerebellum as previously described must not immediately translate into structural cerebellar alteration. Instead, our data suggest, that the temporal onset of the emergence of cerebellar structural changes could be potentially different from other cortical areas such as M1, PMC, and DLPFC. Maybe extensive training over several weeks or years is required to induce such structural changes in the cerebellum 
as previously shown in a cross-sectional study between musicians and non-musicians (Gaser and Schlaug, 2003a).

Consistent with the role of the cerebellum in procedural learning, we here demonstrated that the initial GM volume in the cerebellum (left crus 2 and right cerebellar lobule VI) correlated with the individual amount of motor skill learning in a SPFT and with learning associated structural changes. In other words, subjects who showed the largest gains in motor performance and largest learning-related structural changes were those with the largest initial GM volume in the cerebellum (left crus 2 and right cerebellar lobule VI). This in turn is in line with a recent study showing that individual variations in the function and structure of specific brain regions such as the cerebellum explain some of the differences in an isometric visuomotor tracking task (Tomassini et al., 2010). The finding is corroborated by the additional result that the initial (baseline) GM volume in the cerebellum correlated with the learning-related structural changes in M1, ventral PMC, and DLPFC. Indeed, functional and structural connectivity studies revealed that cerebellar lobule VI has strong connectivity with $\mathrm{M} 1$, whereas DLPFC has strong connections with cerebellar crus 2 that sub-serves motor function (Ramnani, 2006; Krienen and Buckner, 2009). Therefore, our results highlight the importance of selective cerebellar-cortical connections for successful motor learning and structural GM changes.

A limitation of the present study is that structural non-invasive brain imaging methods cannot identify the underlying neurophysiological mechanisms of such bidirectional brain alterations. Thus, animal models have to be conducted to understand how and why inter-individual differences in behavior and learning are related to brain anatomy. Since structural GM alterations were detected already after 5 days of short-term motor skill practice, neurogenesis as the sole underlying mechanisms seems rather unlikely (Draganski and May, 2008). Instead, changes as well as differences in cell size, intracortical myelin, intracortical remodeling of dendritic spines, formation and/or elimination of synapses and/or axonal sprouting seem more likely candidate mechanisms (Trachtenberg et al., 2002; Xu et al., 2009). Furthermore, individual differences in synaptic and/or neuronal pruning processes during development might also play a crucial role for the differential outcome of learning-related GM volume alterations (Kanai

\section{REFERENCES}

Ashburner, J. (2007). A fast diffeomorphic image registration algorithm. Neuroimage 38, 95-113.

Bezzola, L., Mérillat, S., Gaser, C., and Jäncke, L. (2011). Training-induced neural plasticity in golf novices. J. Neurosci. 31, 12444-12448.

Black, J. E., Isaacs, K. R., Anderson, B. J., Alcantara, A. A., and Greenough, W. T. (1990). Learning causes synaptogenesis, whereas motor activity causes angiogenesis, in cerebellar cortex of adult rats. Proc. Natl. Acad. Sci. U.S.A. 87, 5568-5572.

Boyke, J., Driemeyer, J., Gaser, C., Buchel, C., and May, A. (2008).

and Rees, 2011). Alternatively, our findings might be related to differences in metabolic demands during motor skill learning which in turn might cause bidirectional structural brain alterations (Black et al., 1990; Isaacs et al., 1992). Apart from these limitations in the interpretation of the underlying learning-related neural mechanisms, the present study highlights the importance of intra-individual learning success for structural brain alterations rather than the "mere practice." Hence, we propose that the general view "to perform a learning task results in an increase in GM volume" cannot be generalized, but rather should be modified to consider task-specificity, learning success, duration of learning, and other, until now, unknown determinants.

Another potential limitation of the present study is the relatively small sample size $(n=13)$. Hence, it is reasonable to assume that the changes in GM structure are spuriously correlated with our behavioral measures. This, however, seems to be rather unlikely due to several reasons. First, our correlation coefficients were quite strong ( $r=0.83$ for all brain areas detected) even with the relatively small number of subjects tested. The brainbehavior correlations (see Figure 3B) were still significant after removing the subject with the highest change in GM volume and SPFT improvement [left M1: $r=0.691 ; p=0.013$; right ventral PMC: $r=0.780 ; p=0.003$; right DLPFC: $r=0.763 ; p=0.004$, $(n=12)]$. Second, we could show that initial GM volume differences in the cerebellum before SPFT training did not only "predict" performance improvements with training but also seem to "predict" the extend of structural plasticity in brain areas where we found correlations with SPFT improvements. Taken together, this line of evidence renders spurious correlations rather unlikely.

Last but not least, we have to acknowledge the fact that we did not introduce a sedative control group in the present study with no training or a comparison group that performed the SPFT with only random sequences for the same amount of time. Hence, we cannot make definite inferences about the effects of the intervention (SPFT training) on structural brain plasticity. Even though a correlation in general cannot provide a causal relationship, the strong correlation between structural brain alterations and behavioral improvements in our study suggest an intuitive brain-behavior interaction. Future studies should be performed to further support our study claims.

Draganski, B., Gaser, C., Busch, V., Schuierer, G., Bogdahn, U., and May, A. (2004). Neuroplasticity: changes in grey matter induced by training. Nature 427, 311-312.

Draganski, B., and May, A. (2008). Training-induced structural changes in the adult human brain. Behav. Brain Res. 192, 137-142.

Driemeyer, J., Boyke, J., Gaser, C., Buchel, C., and May, A. (2008). Changes in gray matter induced by learningrevisited. PLOS ONE 3:e2669. doi: 10.1371/journal.pone.0002669

Filippi, M., Ceccarelli, A., Pagani, E., Gatti, R., Rossi, A., Stefanelli, L., Falini, A., Comi, G., and Rocca, M
A. (2010). Motor learning in healthy humans is associated to gray matter changes: a tensor-based morphometry study. PLoS ONE 5:e10198. doi: 10.1371/journal.pone.0010198

Fink, G. R., Marshall, J. C., Halligan, P. W., Frith, C. D., Driver, J., Frackowiak, R. S., and Dolan, R. J. (1999). The neural consequences of conflict between intention and the senses. Brain 122 (Pt 3), 497-512.

Floyer-Lea, A., and Matthews, P. M. (2004). Changing brain networks for visuomotor control with increased movement automaticity. J. Neurophysiol. 92, 2405-2412.

to spatiotemporal complexity and cycling frequency. Neuroimage 21, 1416-1427.
Floyer-Lea, A., and Matthews, P. M. (2005). Distinguishable brain 
activation networks for short- and long-term motor skill learning. J. Neurophysiol. 94, 512-518.

Gaser, C., and Schlaug, G. (2003a). Brain structures differ between musicians and non-musicians. J. Neurosci. 23, 9240-9245.

Gaser, C., and Schlaug, G. (2003b). Gray matter differences between musicians and nonmusicians. Ann. N.Y. Acad. Sci. 999, 514-517.

Ghilardi, M., Ghez, C., Dhawan, V., Moeller, J., Mentis, M., Nakamura, T., Antonini, A., and Eidelberg, D. (2000). Patterns of regional brain activation associated with different forms of motor learning. Brain Res. 871, 127-145.

Granert, O., Peller, M., Jabusch, H. C., Altenmuller, E., and Siebner, $\mathrm{H}$. R. (2011). Sensorimotor skills and focal dystonia are linked to putaminal grey-matter volume in pianists. J. Neurol. Neurosurg. Psychiatry 82, 1225-1231.

Halsband, U., and Lange, R. K. (2006). Motor learning in man: a review of functional and clinical studies. J. Physiol. Paris 99, 414-424.

Hikosaka, O., Nakamura, K., Sakai, K., and Nakahara, H. (2002). Central mechanisms of motor skill learning. Curr. Opin. Neurobiol. 12, 217-222.

Hoekzema, E., Carmona, S., RamosQuiroga, J. A., Barba, E., Bielsa, A., Tremols, V., Rovira, M., Soliva, J. C., Casas, M., Bulbena, A., Tobeña, A., and Vilarroya, O. (2011). Traininginduced neuroanatomical plasticity in ADHD: a tensor-based morphometric study. Hum. Brain Mapp. 32, 1741-1749.

Isaacs, K. R., Anderson, B. J., Alcantara, A. A., Black, J. E., and Greenough, W. T. (1992). Exercise and the brain: angiogenesis in the adult rat cerebellum after vigorous physical activity and motor skill learning. J. Cereb. Blood Flow Metab. 12, 110-119.

Jenkins, I. H., Brooks, D. J., Nixon, P. D., Frackowiak, R. S., and Passingham, R. E. (1994). Motor sequence learning: a study with positron emission tomography. J. Neurosci. 14, 3775-3790.

Jueptner, M., Frith, C. D., Brooks, D. J., Frackowiak, R. S., and Passingham, R. E. (1997). Anatomy of motor learning. II. Subcortical structures and learning by trial and error. J. Neurophysiol. 77, 1325-1337.

Jueptner, M., and Weiller, C. (1998). A review of differences between basal ganglia and cerebellar control of movements as revealed by functional imaging studies. Brain 121 (Pt 8), 1437-1449.

Kanai, R., and Rees, G. (2011). The structural basis of inter-individual differences in human behaviour and cognition. Nat. Rev. Neurosci. 12, 231-242.

Klein, A., Andersson, J., Ardekani, B. A., Ashburner, J., Avants, B., Chiang, M. C., Christensen, G. E., Collins, D. L., Gee, J., Hellier, P., Song, J. H., Jenkinson, M., Lepage, C., Rueckert, D., Thompson, P., Vercauteren, T., Woods, R. P., Mann, J. J., and Parsey, R. V. (2009). Evaluation of 14 nonlinear deformation algorithms applied to human brain MRI registration. Neuroimage 46, 786-802.

Krienen, F. M., and Buckner, R. L. (2009). Segregated fronto-cerebellar circuits revealed by intrinsic functional connectivity. Cereb. Cortex 19, 2485-2497.

Landi, S. M., Baguear, F., and DellaMaggiore, V. (2011). One week of motor adaptation induces structural changes in primary motor cortex that predicts long-term memory one year later. J. Neurosci. 31, 11808-11813.

May, A., Hajak, G., Ganssbauer, S., Steffens, T., Langguth, B., Kleinjung, T., and Eichhammer, P. (2007). Structural brain alterations following 5 days of intervention: dynamic aspects of neuroplasticity. Cereb. Cortex 17, 205-210.

Mayka, M. A., Corcos, D. M., Leurgans, S. E., and Vaillancourt, D. E. (2006). Three-dimensional locations and boundaries of motor and premotor cortices as defined by functional brain imaging: a meta-analysis. Neuroimage 31, 1453-1474.

Oldfield, R. C. (1971). The assessment and analysis of handedness: the Edinburgh inventory. Neuropsychologia 9, 97-113.

Ramnani, N. (2006). The primate cortico-cerebellar system: anatomy and function. Nat. Rev. Neurosci. 7, 511-522.

Sadato, N., Campbell, G., Ibanez, V., Deiber, M., and Hallett, M. (1996). Complexity affects regional cerebral blood flow change during sequential finger movements. J. Neurosci. 16, 2691-2700.

Scholz, J., Klein, M. C., Behrens, T. E., and Johansen-Berg, H. (2009).
Training induces changes in whitematter architecture. Nat. Neurosci. 12, 1370-1371.

Schubotz, R. I., and von Cramon, D. Y. (2001a). Functional organization of the lateral premotor cortex: fMRI reveals different regions activated by anticipation of object properties, location and speed. Brain Res. Cogn. Brain Res. 11, 97-112.

Schubotz, R. I., and von Cramon, D. Y. (2001b). Interval and ordinal properties of sequences are associated with distinct premotor areas. Cereb. Cortex 11, 210-222.

Stein, M., Federspiel, A., Koenig, T., Wirth, M., Strik, W., Wiest, R. Brandeis, D., and Dierks, T. (2012). Structural plasticity in the language system related to increased second language proficiency. Cortex 48 , 458-465.

Tanaka, Y., Fujimura, N., Tsuji, T., Maruishi, M., Muranaka, H., and Kasai, T. (2009). Functional interactions between the cerebellum and the premotor cortex for error correction during the slow rate force production task: an fMRI study. Exp. Brain Res. 193, 143-150.

Taubert, M., Draganski, B., Anwander, A., Muller, K., Horstmann, A. Villringer, A., and Ragert, P. (2010). Dynamic properties of human brain structure: learning-related changes in cortical areas and associated fiber connections. J. Neurosci. 30, 11670-11677.

Tegenthoff, M., Ragert, P., Pleger, B., Schwenkreis, P., Forster, A. F., Nicolas, V., and Dinse, H. R. (2005) Improvement of tactile discrimination performance and enlargement of cortical somatosensory maps after $5 \mathrm{~Hz}$ rTMS. PLoS Biol. 3:e362. doi: 10.1371/journal.pbio.0030362

Tomassini, V., Jbabdi, S., Kincses, Z T., Bosnell, R., Douaud, G., Pozzilli, C., Matthews, P. M., and JohansenBerg, H. (2010). Structural and functional bases for individual differences in motor learning. Hum. Brain Mapp. 32, 494-508.

Toni, I., and Passingham, R. E. (1999) Prefrontal-basal ganglia pathways are involved in the learning of arbitrary visuomotor associations: a PET study. Exp. Brain Res. 127, 19-32.

Tost, H., Braus, D. F., Hakimi, S. Ruf, M., Vollmert, C., Hohn, F. and Meyer-Lindenberg, A. (2010). Acute D2 receptor blockade induces rapid, reversible remodeling in human cortical-striatal circuits. Nat. Neurosci. 13, 920-922.

Trachtenberg, J. T., Chen, B. E., Knott, G. W., Feng, G., Sanes, J. R., Welker, E., and Svoboda, K. (2002). Longterm in vivo imaging of experiencedependent synaptic plasticity in adult cortex. Nature 420, 788-794.

Vollmann, H., Conde, V., Sewerin, S., Taubert, M., Sehm, B., Witte, O. W., Villringer, A., and Ragert, P. (2012). Anodal transcranial direct current stimulation (tDCS) over supplementary motor area (SMA) but not pre-SMA promotes short-term visuomotor learning. Brain Stimul. (in press).

Werner, S., Bock, O., Gizewski, E. R., Schoch, B., and Timmann, D. (2010). Visuomotor adaptive improvement and aftereffects are impaired differentially following cerebellar lesions in SCA and PICA territory. Exp. Brain Res. 201, 429-439.

Xu, T., Yu, X., Perlik, A. J., Tobin, W. F., Zweig, J. A., Tennant, K., Jones, T., and Zuo, Y. (2009). Rapid formation and selective stabilization of synapses for enduring motor memories. Nature 462, 915-919.

Yang, G., Pan, F., and Gan, W. B. (2009). Stably maintained dendritic spines are associated with lifelong memories. Nature 462, 920-924.

Conflict of Interest Statement: The authors declare that the research was conducted in the absence of any commercial or financial relationships that could be construed as a potential conflict of interest.

Received: 13 March 2012; accepted: 02 May 2012; published online: 16 May 2012.

Citation: Gryga M, Taubert M, Dukart $J$, Vollmann $H$, Conde V, Sehm B, Villringer $A$ and Ragert $P$ (2012) Bidirectional gray matter changes after complex motor skill learning. Front. Syst. Neurosci. 6:37. doi: 10.3389/fnsys. 2012.00037

Copyright (C) 2012 Gryga, Taubert, Dukart, Vollmann, Conde, Sehm, Villringer and Ragert. This is an openaccess article distributed under the terms of the Creative Commons Attribution Non Commercial License, which permits non-commercial use, distribution, and reproduction in other forums, provided the original authors and source are credited. 